TRANSACTIONS OF THE

AMERICAN MATHEMATICAL SOCIETY

Volume 361, Number 8, August 2009, Pages 4471-4489

S 0002-9947(09)04819-3

Article electronically published on March 10, 2009

\title{
LOG CANONICAL MODELS FOR THE MODULI SPACE OF CURVES: THE FIRST DIVISORIAL CONTRACTION
}

\author{
BRENDAN HASSETT AND DONGHOON HYEON
}

\begin{abstract}
In this paper, we initiate our investigation of log canonical models for $\left(\overline{\mathcal{M}}_{g}, \alpha \delta\right)$ as we decrease $\alpha$ from 1 to 0 . We prove that for the first critical value $\alpha=9 / 11$, the $\log$ canonical model is isomorphic to the moduli space of pseudostable curves, which have nodes and cusps as singularities. We also show that $\alpha=7 / 10$ is the next critical value, i.e., the log canonical model stays the same in the interval $(7 / 10,9 / 11]$. In the appendix, we develop a theory of log canonical models of stacks that explains how these can be expressed in terms of the coarse moduli space.
\end{abstract}

\section{INTRODUCTION}

This article is the first of a series of papers investigating the birational geometry of the moduli space $\bar{M}_{g}$ of stable curves of genus $g$. The guiding problem is to understand the canonical model of $\bar{M}_{g}$,

$$
\operatorname{Proj}\left(\bigoplus_{n \geq 0} \Gamma\left(\bar{M}_{g}, n K_{\bar{M}_{g}}\right)\right) \text {, }
$$

when $\bar{M}_{g}$ is of general type. The moduli space is known to be of general type for $g \geq 24$ by work of Eisenbud, Harris, and Mumford HM82; recently, Farkas has announced results for $g=22$ [Far06].

It is a fundamental conjecture of birational geometry (recently proven by Birkar, Cascini, Hacon and $\mathrm{M}^{\mathrm{c}}$ Kernan [BCHM06]) that canonical rings of varieties of general type are finitely generated. The projective variety associated with this graded ring is called the canonical model. Explicit canonical models of $\bar{M}_{g}$ are not known for any genus $g$. To get around this difficulty, we study the intermediate log canonical models

$$
\bar{M}_{g}(\alpha):=\operatorname{Proj}\left(\bigoplus_{n \geq 0} \Gamma\left(n\left(K_{\overline{\mathcal{M}}_{g}}+\alpha \delta\right)\right)\right)
$$

for various values of $\alpha \in \mathbb{Q} \cap[0,1]$. Here $\overline{\mathcal{M}}_{g}$ is the moduli stack, $\delta_{i}, i=0, \ldots,\lfloor g / 2\rfloor$, its boundary divisors, and $K_{\overline{\mathcal{M}}_{g}}$ its canonical divisor. Let $\overline{\mathcal{M}}_{g} \rightarrow \bar{M}_{g}$ denote the morphism to the coarse moduli space, which has boundary divisors $\Delta_{i}$ and canonical class $K_{\bar{M}_{g}}$.

Received by the editors November 28, 2007.

2000 Mathematics Subject Classification. Primary 14E30, 14H10.

(C)2009 American Mathematical Society Reverts to public domain 28 years from publication 
A general discussion of log canonical models for stacks is offered in Appendix A. but we sketch the basic idea here. Assume that $g \geq 4$; the $g=2$ case is discussed in Has05]. Then the $\mathbb{Q}$-divisor on the coarse moduli space

$$
K_{\bar{M}_{g}}+\alpha\left(\Delta_{0}+\Delta_{2}+\ldots+\Delta_{\lfloor g / 2\rfloor}\right)+\frac{1+\alpha}{2} \Delta_{1}
$$

pulls back to $K_{\overline{\mathcal{M}}_{g}}+\alpha \delta$ on the moduli stack (see Remark 4.9 of [Has05]). We shall often abuse notation by conflating these divisors. The universal property of the coarse moduli space implies that sections of invertible sheaves on $\overline{\mathcal{M}}_{g}$ are all pullbacks of sections of the corresponding reflexive sheaves on $\bar{M}_{g}$. The log canonical model of $\overline{\mathcal{M}}_{g}$ with respect to $K_{\overline{\mathcal{M}}_{g}}+\alpha \delta$ can thus be identified with the log canonical model of $\bar{M}_{g}$ with respect to $(\dagger)$ (see Proposition A.13).

For large values of $\alpha$ these log canonical models are well-understood. Results of Mumford, Cornalba, and Harris imply that $\bar{M}_{g}(\alpha) \simeq \bar{M}_{g}$ if and only if $9 / 11<\alpha \leq$ 1. Indeed, by the uniqueness of log canonical models, it suffices to show the pair $\left(\overline{\mathcal{M}}_{g}, \alpha \delta\right)$ has $\log$ canonical singularities and $K_{\overline{\mathcal{M}}_{g}}+\alpha \delta$ is ample on $\bar{M}_{g}$, for $\alpha$ in this range. By Theorem 2 of [HM82], $\bar{M}_{g} \backslash \Delta_{1}$ actually has canonical singularities; the pair $\left(\overline{\mathcal{M}}_{g}, \alpha \delta\right)$ is a smooth stack with a normal-crossings divisor and thus is log canonical. The main result of [CH88] says that

$$
a \lambda-\delta=\frac{a}{13}\left(K_{\overline{\mathcal{M}}_{g}}+\left(2-\frac{13}{a}\right) \delta\right)
$$

is ample for $a>11$. Indeed, $\left(\overline{\mathcal{M}}_{g}, \alpha \delta\right)$ is a strict log canonical model in the sense that all divisors with negative discrepancies lie over $\delta$ (cf. Has05], §4.3).

In this paper, we describe what happens when $\alpha=9 / 11$. The pair $\left(\overline{\mathcal{M}}_{g}, 9 / 11 \delta\right)$ remains $\log$ terminal, but $K_{\overline{\mathcal{M}}_{g}}+9 / 11 \delta$ is not ample on $\bar{M}_{g}$. Mumford [Mum77] described an extremal ray $R$ for this divisor, parametrizing the elliptic tails. Recall that the normalization of $\delta_{1}$ is isomorphic to $\overline{\mathcal{M}}_{1,1} \times \overline{\mathcal{M}}_{g-1,1}$ and $\bar{M}_{1,1} \simeq \mathbb{P}^{1}$. Our ray is the class of the fiber under projection to the second factor. Explicitly, for each $\left(C_{2}, p\right)$ of $\bar{M}_{g-1,1}$ consider the stable curves $C=C_{1} \cup_{p} C_{2}$, where $\left(C_{1}, p\right)$ is a varying curve in $\bar{M}_{1,1} \simeq \mathbb{P}^{1}$, meeting $C_{2}$ at a single node $p$. These are parametrized by a rational curve $R\left(C_{2}, p\right)$; the class $R=\left[R\left(C_{2}, p\right)\right]$ is independent of $C_{2}$ and $p$ and

$$
\left(K_{\overline{\mathcal{M}}_{g}}+9 / 11 \delta\right) \cdot R=0 .
$$

This is the unique extremal ray for $K_{\overline{\mathcal{M}}_{g}}+9 / 11 \delta$; it meets every other curve class in $\bar{M}_{g}$ positively (by the analysis of [CH88] or $\S 6$ of GKM02]).

Applying the basepoint-freeness theorem to $K_{\overline{\mathcal{M}}_{g}}+9 / 11 \delta$ (see Corollary A.14), we obtain an extremal contraction of $R$,

$$
\Upsilon: \bar{M}_{g} \rightarrow \bar{M}_{g}(9 / 11)
$$

Since $R . \Delta_{1}<0, \Upsilon$ is birational with exceptional locus contained in $\Delta_{1}$. More precisely, its restriction to the generic point of $\Delta_{1}$ is a projection onto the $\bar{M}_{g-1,1}$ factor. Thus $\Upsilon$ is a divisorial contraction, and $\bar{M}_{g}(9 / 11)$ remains $\mathbb{Q}$-factorial.

Is $\bar{M}_{g}(9 / 11)$ the coarse moduli space of some moduli stack of curves with prescribed properties? Such a moduli functor is implicit in the work of D. Schubert Sch91. He constructed the moduli space $\bar{M}_{g}^{p s}$ of pseudostable curves applying geometric invariant theory (GIT) to tricanonical curves. Recall that a cusp is a 
curve singularity analytically-locally isomorphic to $y^{2}=x^{3}$. A complete curve is pseudostable if

(1) it is connected, reduced, and has only nodes and cusps as singularities;

(2) every subcurve of genus one meets the rest of the curve in at least two points;

(3) the canonical sheaf of the curve is ample.

The last condition means that each subcurve of genus zero meets the rest of the curve in at least three points. The corresponding moduli stack is denoted $\overline{\mathcal{M}}_{g}^{p s}$.

Theorem 1.1. There is a morphism of stacks

$$
\mathcal{T}: \overline{\mathcal{M}}_{g} \rightarrow \overline{\mathcal{M}}_{g}^{p s}
$$

which is an isomorphism in the complement of $\delta_{1}$. For a stable curve $C \in \delta_{1}, \mathcal{T}(C)$ is obtained by replacing each elliptic tail of $C$ with a cusp. $\bar{M}_{g}^{p s}$ and $\bar{M}_{g}(9 / 11)$ are isomorphic compactifications of the moduli space of curves, and the induced morphism on coarse moduli spaces

$$
T: \bar{M}_{g} \rightarrow \bar{M}_{g}^{p s}
$$

coincides with the extremal contraction $\Upsilon$.

See 93 for a precise formulation of what it means to replace an elliptic tail with a cusp. We can characterize the $\alpha$ for which $\bar{M}_{g}(\alpha)$ is isomorphic to the moduli space of pseudostable curves:

Theorem 1.2. For $7 / 10<\alpha \leq 9 / 11, \bar{M}_{g}(\alpha)$ exists as a projective variety and is isomorphic to $\bar{M}_{g}^{p s}$.

We shall describe what happens at the next critical value $\alpha=7 / 10$ in our forthcoming paper [HH08].

Finally, we survey recent results of a similar flavor. Shepherd-Barron has analyzed the canonical model of the moduli space of principally polarized abelian varieties of dimension $\geq 12$ [SB06, Theorem 0.2]. Hacking, Keel, and Tevelev have considered log canonical models for moduli of hyperplane arrangements [KT06, 1.6] (cf. HKT06). These can be understood explicitly in special cases, e.g., $\left(\bar{M}_{0, n}, \delta\right)$ is a $\log$ canonical model Has03, 7.1].

Throughout, we work over an algebraically closed field $k$ of characteristic zero.

\section{Moduli stack of Pseudostable CuRves}

To lay groundwork for the construction of $T: \bar{M}_{g} \rightarrow \bar{M}_{g}^{p s}$ in the subsequent section, we shall sketch Schubert's construction of the moduli space of pseudostable curves Sch91 and reformulate the main results using language of stacks. Throughout this section we assume $g>2$. (The special features of the $g=2$ case are addressed in [HL07.)

Definition 2.1. An $n$-canonical curve

$$
C \subset \mathbb{P}^{N}, \quad N:= \begin{cases}g-1 & \text { if } n=1, \\ (2 n-1)(g-1)-1 & \text { if } n>1\end{cases}
$$

is a Deligne-Mumford stable curve imbedded by the sections of the pluricanonical bundle $\omega_{C}^{\otimes n}$. ( $\omega_{C}^{\otimes n}$ is very ample for $n \geq 3$ [DM69, Theorem 1.2].) 
These form a locally closed subset Chow $_{n}$ of the Chow variety parametrizing curves in $\mathbb{P}^{N}$ of degree $d:=2 n(g-1)$. Schubert investigated the stability of points in Chow $_{3}$. He proves that a Chow point is GIT stable if and only if the corresponding cycle is a pseudostable curve of genus $g$. Furthermore, there are no strictly semistable points. Here is the idea of the argument: Using the methods of [Mum77] for finding destabilizing one-parameter subgroups, one checks that the cycles associated to nonpseudostable curves are necessarily GIT unstable. The proof that pseudostable curves have stable Chow points is indirect: By general results from Mum77, smooth tricanonical curves have GIT stable Chow points. Let $C \subset \mathbb{P}^{5 g-6}$ be a tricanonically-embedded pseudostable curve. A deformationtheoretic argument shows there exists a flat family of curves in $\mathbb{P}^{5 g-6}$

$$
\pi: \mathcal{C} \rightarrow \Delta=\text { Spec } k[[t]]
$$

with closed fiber $C$ and generic fiber smooth and tricanonically embedded. Consider the induced morphism to the GIT quotient

$$
\mu: \Delta \rightarrow \text { Chow }_{3} / / \mathrm{SL}_{5 g-5}
$$

of course, $\mu(0)$ must correspond to certain semistable points in Chow Ch $_{3}$ After a base change $\widetilde{\Delta} \rightarrow \Delta$ (substitute $t=\tau^{M}$ for some $M$ ), we obtain a lift

$$
\widetilde{\mu}: \widetilde{\Delta} \rightarrow \text { Chow }_{3}^{s s}
$$

into the semistable locus of the Chow variety. The generic point maps into the stable locus. Let $\mathcal{D} \rightarrow \widetilde{\Delta}$ be the family of pseudostable curves associated to $\widetilde{\mu}$. Note that $\mathcal{D}$ and the pull-back $\widetilde{\mathcal{C}}:=\mathcal{C} \times_{\Delta} \widetilde{\Delta}$ agree up to the action of $\mathrm{SL}_{5(g-1)}$. To prove that $C$ is semistable, we show that the closed fibers of $\mathcal{C}$ and $\mathcal{D}$ agree. We require the following uniqueness result, which may be regarded as the valuative criterion for separatedness for the moduli functor of pseudostable curves:

Proposition 2.2 (cf. Lemma 4.2 of [Sch91]). Let $\mathcal{D}_{1}, \mathcal{D}_{2} \rightarrow \Delta$ be flat families of pseudostable curves with smooth generic fibers, isomorphic over $k((t))$. Then the closed fibers are isomorphic as well.

Finally, suppose there were strictly semistable points: Then some point of Chow $_{3}^{\text {ss }}$ would have a positive-dimensional stabilizer, but pseudostable curves have finite automorphism groups [Sch91, pp. 312].

Implicit in Schubert's work is the moduli stack $\overline{\mathcal{M}}_{g}^{\text {ps }}$ of pseudostable curves:

$$
\overline{\mathcal{M}}_{g}^{p s}(S)=\left\{\begin{array}{l|l}
f: C \rightarrow S & \begin{array}{l}
\text { (i) } f \text { is a proper and flat morphism, } \\
\text { (ii) geometric fibres of } f \text { are pseudo- } \\
\text { stable curves of genus } g .
\end{array}
\end{array}\right\}
$$

where $S$ is a scheme of finite type over $k$. This is a Deligne-Mumford stack, as pseudostable curves have finite automorphism groups when $g>2$. Its coarse moduli space is denoted $\bar{M}_{g}^{p s}$.

Theorem 2.3 (cf. SSch91, Theorem 5.4]). The quotient stack $\left[\mathbf{C h o w}_{3}^{\text {s }} / \mathrm{PGL}_{5 g-5}\right]$ is isomorphic to $\overline{\mathcal{M}}_{g}^{p s}$ and

$$
\mathrm{Chow}_{3} / / \mathrm{SL}_{5 g-5} \simeq \bar{M}_{g}^{p s} \text {. }
$$

Remark 2.4. It is shown in Mor08] that most of Schubert's analysis goes through in the four-canonical case except that curves with elliptic tails as well as the corresponding cuspidal curves (Proposition 3.1) are strictly Chow semistable and are 
identified in the quotient space $\mathbf{C h o w}_{4} / / \mathrm{SL}_{7 g-7}$. Although this is isomorphic to the coarse moduli space of $\overline{\mathcal{M}}_{g}^{p s}$, the quotient stack $\left[\mathbf{C h o w}_{4}^{s s} / \mathrm{PGL}_{7 g-7}\right]$ is not separated.

When the Hilbert scheme $\mathbf{H i l b}_{4}$ (with asymptotic linearization [HH08]) is used in place of the Chow variety, a four-canonical curve is Hilbert stable if and only if it is pseudostable, and there are no strictly Hilbert semistable points. Hence the quotient stack $\left[\mathbf{H i l b}_{4}^{s} / \mathrm{PGL}_{7 g-7}\right]$ is isomorphic to $\overline{\mathcal{M}}_{g}^{p s}$ and $\mathbf{H i l b}_{4} / / \mathrm{SL}_{7 g-7}$ is isomorphic to the coarse moduli space $\bar{M}_{g}^{p s}$.

\section{Constructing the natural transformation $\mathcal{T}$}

Here we prove the first part of Theorem 1.1. Throughout, we assume $g>2$.

\subsection{Replacing elliptic tails with cusps.}

Proposition 3.1. Let $C$ be a stable curve of genus $g>2$ with elliptic tails $E_{1}, \ldots, E_{r}$, i.e., connected genus-one subcurves meeting the rest of $C$ in a single node. Let $D$ denote the union of the components of $C$ other than elliptic tails and $p_{i}$ the node where $E_{i}$ meets $D, i=1, \ldots, r$. Then there exists a unique curve $\mathcal{T}(C)$ characterized by the following properties:

(1) there is a birational morphism $\nu: D \rightarrow \mathcal{T}(C)$, which is an isomorphism away from $p_{1}, \ldots, p_{r}$;

(2) $\nu$ is bijective and maps each $p_{i} \in D$ to a cusp $q_{i} \in \mathcal{T}(C)$, formally isomorphic to $y^{2}=x^{3}$.

There is a unique replacement morphism $\xi_{C}: C \rightarrow \mathcal{T}(C)$ with $\left.\xi_{C}\right|_{D}=\nu$ and $\left.\xi_{C}\right|_{E_{i}}$ constant. Note that $\mathcal{T}(C)$ has arithmetic genus $g$.

Proof. To determine $\mathcal{T}(C)$, it suffices to specify the subrings

$$
\mathcal{O}_{\mathcal{T}(C), q_{i}} \subset \mathcal{O}_{D, p_{i}} .
$$

Let $\mathfrak{m}_{D, p_{i}} \subset \mathcal{O}_{D, p_{i}}$ be the maximal ideal and define $\mathcal{O}_{\mathcal{T}(C), q_{i}}$ to be the algebra generated by the constants and $\mathfrak{m}_{D, p_{i}}^{2}$. A local computation shows that $\mathcal{T}(C)$ has a cusp at $q_{i}$. Indeed, $\mathfrak{m}_{\mathcal{T}(C), q_{i}}$ is generated by two elements $\hat{x}$ and $\hat{y}$, vanishing at $p_{i}$ to orders two and three respectively, whence $\hat{y}^{2}=c \hat{x}^{3}$ for some unit $c \in \mathcal{O}_{D, p_{i}}$. Conversely, any germ of a cuspidal curve normalized by $\left(D, p_{i}\right)$ is obtained in this way. The morphism $\nu$ is the normalization of the cusps $q_{1}, \ldots, q_{r}$.

A morphism of stacks

$$
\mathcal{T}: \overline{\mathcal{M}}_{g} \rightarrow \overline{\mathcal{M}}_{g}^{p s}
$$

assigns to each stable curve $f: C \rightarrow S$ (over a scheme $S$ of finite type) a pseudostable curve $\mathcal{T}(f): \mathcal{T}(C) \rightarrow S$. This recipe must be compatible with base extension and isomorphisms. (This is just the definition of a 1-morphism between two categories fibered in groupoids over the category of schemes; cf. LMB00, chapter 2].)

Our main tool is a replacement morphism $\xi_{C}: C \rightarrow \mathcal{T}(C)$ defined over $S$, with the following properties:

(a) over the open subset $S_{0} \subset S$ mapping to the complement to $\delta_{1}, \xi_{C}$ is an isomorphism; 
(b) for $s \in S$ mapped to $\delta_{1}, \xi_{C_{s}}: C_{s} \rightarrow \mathcal{T}(C)_{s}$ replaces each elliptic tail in $C_{s}$ with a cusp;

(c) $\xi_{C}$ is compatible with base extension and isomorphisms as indicated above.

We shall specify $\mathcal{T}$ and $\xi$ using a suitable atlas of $\overline{\mathcal{M}}_{g}$ in the smooth or flat topology. Let $\pi: Z \rightarrow U$ be a stable curve over a scheme so that the classifying map $\rho_{\pi}: U \rightarrow \overline{\mathcal{M}}_{g}$ is faithfully flat. For example, we could take $U$ to be $\mathbf{C h o w}_{3}^{s}$ (or the corresponding Hilbert scheme) and $\pi: Z \rightarrow U$ the universal family. Let $R \rightrightarrows$ $U$ be the corresponding presentation of $\overline{\mathcal{M}}_{g}$, where $R$ encodes the isomorphisms among the fibers of $\pi$. To construct $\mathcal{T}$ and $\xi$ over $\overline{\mathcal{M}}_{g}$, it suffices to construct $\mathcal{T}(\pi): \mathcal{T}(Z) \rightarrow U$ and $\xi_{Z}$ compatibly with the isomorphism relation $R$. Indeed, given an arbitrary stable curve $f: C \rightarrow S$ with classifying map $\rho_{f}: S \rightarrow \overline{\mathcal{M}}_{g}$, we can pull back to the fiber product

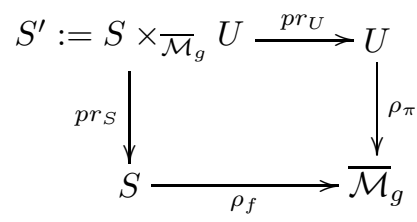

Consider the resulting stable curves

$$
p r_{S}^{*} f: p r_{S}^{*} C \rightarrow S^{\prime}, \quad p r_{U}^{*} \pi: p r_{U}^{*} Z \rightarrow S^{\prime},
$$

with classifying maps $\rho_{f} \circ p r_{S}$ and $\rho_{\pi} \circ p r_{U}$. The commutativity of the fiber product diagram implies that our two stable curves are isomorphic over $S^{\prime}$. By basechange, we have

$$
p r_{U}^{*} \mathcal{T}(\pi): p r_{U}^{*} \mathcal{T}(Z) \rightarrow S^{\prime}, \quad p r_{U}^{*} \xi_{Z}: p r_{U}^{*} Z \rightarrow p r_{U}^{*} \mathcal{T}(Z),
$$

and thus the corresponding constructions for $p r_{S}^{*} f: p r_{S}^{*} C \rightarrow S^{\prime}$. Now $p r_{S}$ is the basechange of $\rho_{\pi}$ and thus is faithfully flat. Since $\mathcal{T}$ is compatible with isomorphisms, the desired

$$
\mathcal{T}(f): \mathcal{T}(C) \rightarrow S, \quad \xi_{C}: C \rightarrow \mathcal{T}(C)
$$

exist by descent.

3.2. Sketch construction of the morphism. Assume $\pi: Z \rightarrow U$ is a stable curve over a smooth base with a faithfully flat classifying map $\rho_{\pi}: U \rightarrow \overline{\mathcal{M}}_{g}$. Let $W=\rho_{\pi}^{*} \delta_{1}$. Let $\mu_{\pi}: Z \rightarrow \overline{\mathcal{M}}_{g, 1}$ denote the classifying morphism to the universal family, $\delta_{1,\{1\}} \subset \overline{\mathcal{M}}_{g, 1}$ the boundary divisor corresponding to the elliptic tails, and $E=\mu_{\pi}^{*} \delta_{1,\{1\}}$, which is also a Cartier divisor on $Z$.

Example 3.2. It may seem counterintuitive that the divisor of elliptic tails should always be Cartier. For example, consider a stable curve

$$
\pi: Z \rightarrow \Delta=\text { Spec } k[[t]]
$$

with smooth generic fiber and special fiber

$$
\pi^{-1}(0)=C_{1} \cup_{p} C_{2},
$$

where $C_{1}$ is smooth of genus one, $C_{2}$ is smooth of genus $g-1$, and $\{p\}=C_{1} \cap C_{2}$ is a single node. If $Z$ has a local analytic equation $x y=t^{m}$ at $p$, then $C_{1}$ is not Cartier at $p$ unless $m=1$. However, note that $E \equiv m C_{1}$ is a Cartier divisor. 
Fix

$$
L=\omega_{\pi}(E),
$$

where $\omega_{\pi}$ is the dualizing sheaf for the morphism $\pi$. Away from $W$ we have $L=\omega_{\pi}$, and for each $n>0, \pi_{*} L^{n}$ is locally free of rank

$$
k_{n}:= \begin{cases}g & \text { if } n=1 \\ (2 n-1)(g-1) & \text { if } n \geq 2,\end{cases}
$$

over $U \backslash W$. Furthermore, the higher-direct image

$$
R^{1} \pi_{*} L^{n} \simeq \begin{cases}\mathcal{O}_{U} & \text { if } n=1, \\ 0 & \text { if } n \geq 2,\end{cases}
$$

over $U \backslash W$. In particular, $\left.\pi_{*} L^{n}\right|_{u} \simeq \Gamma\left(Z_{u}, \omega_{Z_{u}}^{n}\right)$ for each $u \in U \backslash W$.

The family $\left\{\left.\pi_{*} L^{n}\right|_{u}\right\}_{u \in U}$ of linear systems over $U$ will induce the $U$-morphism $Z \rightarrow \mathcal{T}(Z)$. For the remainder of this section, we focus our attention on the behavior of these linear systems near a point $u_{0} \in W$. In $\$ 3.5$ we shall prove the local freeness of $\pi_{*} L^{n}$. The proof depends on identifying the limits of linear series $\left.\pi_{*} L^{n}\right|_{u}$, which can be obtained by using CF91. We do this in $\$ 3.4$ and also show that each linear system $\left.\pi_{*} L^{n}\right|_{u}$ replaces the elliptic tails of $Z_{u}$ with cusps.

3.3. An explicit complex computing the cohomology of $L$. Suppose that $Z_{u_{0}}:=C$ has $r$ elliptic tails $E_{1}, \ldots, E_{r}$, and let $D$ denote the union of the remaining components. In analyzing whether $\pi_{*} L^{n}$ is locally free, we may liberally apply faithfully flat base-extensions $U^{\prime} \rightarrow U$. (Cohomology commutes with flat basechange, and being locally free is a local property in the faithfully flat topology.) For simplicity, we will not use new notation for these base extensions.

After such a base extension, we may assume there exist sections $s_{1}, \ldots, s_{r}: U \rightarrow$ $Z$ so that $s_{i}\left(u_{0}\right) \in E_{i}$ as a smooth point of $Z_{u_{0}}$. Over this neighborhood, we consider the exact sequence

$$
\left.0 \rightarrow L^{n} \rightarrow L^{n}\left(s_{1}+\ldots+s_{r}\right) \rightarrow L^{n}\left(s_{1}+\ldots+s_{r}\right)\right|_{s_{1}, \ldots, s_{r}} \rightarrow 0 .
$$

The last term is supported in $s_{1}\left(u_{0}\right), \ldots, s_{r}\left(u_{0}\right)$, a finite set, and thus has vanishing higher cohomology. As for the second term,

$$
\begin{aligned}
& H^{1}\left(Z_{u_{0}}, L^{n}\left(s_{1}+\ldots+s_{r}\right) \mid Z_{u_{0}}\right) \\
& \quad=H^{1}\left(D,\left.L^{n}\right|_{D}\right) \oplus\left(\bigoplus_{i=1}^{r} H^{1}\left(E_{i}, \mathcal{O}_{E_{i}}\left(s_{i}\left(u_{0}\right)\right)\right)\right)=0 .
\end{aligned}
$$

The resulting long exact sequence takes the form

$$
0 \rightarrow \pi_{*} L^{n} \rightarrow F^{0} \stackrel{\varphi}{\rightarrow} F^{1} \rightarrow R^{1} \pi_{*} L^{n} \rightarrow 0
$$

with $F^{0}:=\pi_{*}\left(L^{n}\left(s_{1}+\ldots+s_{r}\right)\right)$ and $F^{1}:=\pi_{*}\left(\left.L^{n}\left(s_{1}+\ldots+s_{r}\right)\right|_{s_{1}, \ldots, s_{r}}\right)$ locally free of rank $r_{0}$ and $r_{1}$, respectively. The cokernel sheaf $Q:=\operatorname{Coker}\left(\pi_{*} L^{n} \rightarrow F^{0}\right)$ is a subsheaf of a locally-free sheaf, hence is locally-free of rank $r_{0}-k_{n}$ away from a subset $Y \subset U$ of codimension $\geq 2$. Note that $Y$ is contained in the locus where $R^{1} \pi_{*} L^{n}$ fails to be locally free, and thus is a subset of $W=\rho_{\pi}^{*} \delta_{1}$. 
3.4. Some results from limit linear series. Let $B$ be spectrum of a DVR over $k$, with special point 0 and closed point $b$. Consider a morphism $\beta:(B, 0) \rightarrow\left(U, u_{0}\right)$ with $\beta(0)=u_{0} \in W$ and $\beta(b) \notin W$. We have the fiber square

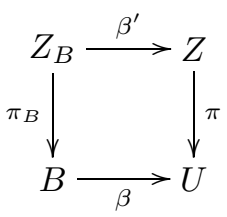

and we write $L_{B}=\beta^{\prime *} L$. The direct image $\pi_{B *} L_{B}^{n}$ is locally free of rank $k_{n}-$ it is a subsheaf of $\beta^{*} F_{0}$.

Proposition 3.3 ([CF91, 4.3.iii]). For $n \geq 1, V_{n}:=\left.\pi_{B *} L_{B}^{n}\right|_{0}$ is naturally identified with

$$
\Gamma\left(D, \omega_{D}^{n}\left((2 n-2) \sum_{j=1}^{r} p_{j}\right)\right)+\left\langle\sigma_{1}^{n}, \ldots, \sigma_{r}^{n}\right\rangle
$$

as a subspace of $H^{0}\left(C,\left.L^{n}\right|_{C}\right)$, where $\sigma_{j}$ are sections of $V_{1}$ such that $\sigma_{j}\left(p_{j}\right) \neq 0$ and $\sigma_{j}$ vanishes to order two at $p_{i}$ for $i \neq j$.

Corollary 3.4. For $n \geq 2$, the linear series $V_{n}$ defines a morphism

$$
C \rightarrow \mathbb{P}^{k_{n}-1}
$$

with image $\mathcal{T}(C)$. The induced $C \rightarrow \mathcal{T}(C)$ is the morphism introduced in Proposition 3.1

Proof. The linear series $V_{n}$ contains

$$
\Gamma\left(D, \omega_{D}^{n}\left((2 n-2) \sum_{j=1}^{r} p_{j}\right)\right) .
$$

For any pointed stable curve $\left(D, p_{1}, \ldots, p_{r}\right),\left(\omega_{D}\left(p_{1}+\ldots+p_{r}\right)\right)^{2}$ is very ample unless $g(D)=2$ and $r=0$, which cannot occur. Thus the sections of $V_{n}$ induce an imbedding of $D$ away from $p_{1}, \ldots, p_{r}$. Proposition 3.3 implies that the vanishing sequence of $V_{n}$ at each $p_{j}$ is $(0,2,3, \ldots)$, and $p_{j}$ is thus mapped to a cusp. Also, the images of $p_{j}$ are distinct, as they are separated by $\sigma_{j}^{n}$. Finally, sections in $V_{n}$ are constant along the $E_{i}$, but not identically zero, so each $E_{i}$ is collapsed to the corresponding cusp.

\subsection{Local freeness of $\pi_{*} L^{n}$.}

Proposition 3.5. For each integer $n \geq 1, \pi_{*} L^{n}$ is locally free of rank $k_{n}$.

Proof. Given a locally-free sheaf $F$ on $U$ of rank $r$, let $\operatorname{Grass}(m, F)$ denote the scheme representing the functor

$$
\underline{\operatorname{Grass}}(m, F): U \text {-schemes } \rightarrow \text { Sets }
$$

that associates to a $U$-scheme $\beta: B \rightarrow U$ the set of rank- $m$ subbundles of $\beta^{*} F$, or equivalently, rank- $(r-m)$ locally-free quotients of $\beta^{*} F$. Our map $\varphi: F^{0} \rightarrow F^{1}$ induces a morphism

$$
\tau: U \backslash Y \rightarrow \operatorname{Grass}\left(r_{0}-k_{n},\left(F^{0}\right)^{*}\right) \times \operatorname{Grass}\left(r_{0}-k_{n}, F^{1}\right)
$$


and a rational map over $Y$. Via the Plücker and Segre embeddings

$$
\begin{aligned}
& \operatorname{Grass}\left(r_{0}-k_{n},\left(F^{0}\right)^{*}\right) \times \operatorname{Grass}\left(r_{0}-k_{n}, F^{1}\right) \hookrightarrow \mathbb{P}\left(\bigwedge^{r_{0}-k_{n}}\left(F^{0}\right)^{*}\right) \times \mathbb{P}\left(\bigwedge^{r_{0}-k_{n}} F^{1}\right) \\
& \hookrightarrow \mathbb{P}\left(\bigwedge^{r_{0}-k_{n}}\left(F^{0}\right)^{*} \otimes \bigwedge^{r_{0}-k_{n}} F^{1}\right)=\mathbb{P}\left(\mathcal{H o m}\left(\bigwedge^{r_{0}-k_{n}} F^{0}, \bigwedge^{r_{0}-k_{n}} F^{1}\right)\right)
\end{aligned}
$$

we consider $\tau$ as a rational map $U \rightarrow \mathbb{P}\left(\mathcal{H o m}\left(\bigwedge^{r_{0}-k_{n}} F^{0}, \bigwedge^{r_{0}-k_{n}} F^{1}\right)\right)$ given by the section $\bigwedge^{r_{0}-k_{n}} \varphi$. The indeterminacy locus of $\tau$ is precisely the zero locus of $\bigwedge^{r_{0}-k_{n}} \varphi$ which is defined by the ideal generated by $\left(r_{0}-k_{n}\right) \times\left(r_{0}-k_{n}\right)$ minors of $\varphi$. This is the $\left(r_{1}-r_{0}+k_{n}\right)$ th Fitting ideal $\mathcal{I}$ of $R^{1} \pi_{*} L^{n}$. Blowing up along $\mathcal{I}$ to resolve indeterminacy, we get

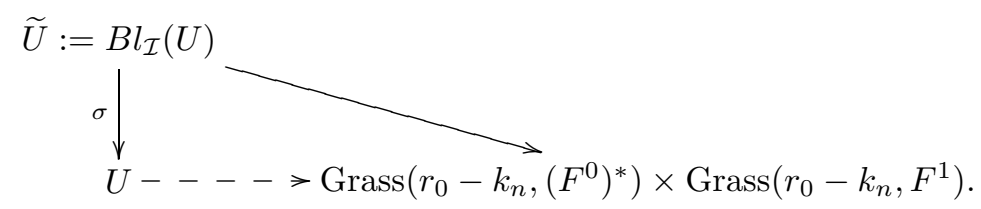

Let $\widetilde{\tau}$ denote the lift of $\tau$ to $\widetilde{U}$. We claim that $\sigma$ is an isomorphism so that $\widetilde{\tau}$ is defined on $U$ and extends $\tau$. This implies that there exists a subbundle $K$ of $F^{0}$ over $U$ such that $\left.\left.K\right|_{U \backslash Y} \simeq \pi_{*} L^{n}\right|_{U \backslash Y}$. For a general discussion of flattening and blowing up, we refer the reader to Ray72, chapter 4].

Since $\sigma$ is proper birational and $U$ is normal, it suffices to show that $\sigma$ is quasifinite. Suppose that it is not quasi-finite and there exist two distinct closed points $x_{1}, x_{2} \in \widetilde{U}$ such that $\tau$ is not regular at $u:=\sigma\left(x_{1}\right)=\sigma\left(x_{2}\right)$. Choose curves $\widetilde{\beta}_{i}: B=\operatorname{Spec} R \rightarrow \widetilde{U}$ with $\widetilde{\beta}_{i}(0)=x_{i}$ and $\widetilde{\beta}_{i}(r) \notin \delta_{1}, i=1,2$. Here $R=k[[t]]$, 0 denotes the closed point and $r$, the generic point. Let $\beta_{i}=\sigma \circ \widetilde{\beta}_{i}$. Due to the functoriality of $\operatorname{Grass}\left(k_{n}, F^{0}\right), \widetilde{\tau} \circ \widetilde{\beta}_{i}$ corresponds to a locally-free quotient $0 \rightarrow$ $K_{i} \rightarrow \beta_{i}^{*} F^{0} \rightarrow Q_{i} \rightarrow 0$ such that $\operatorname{rank}\left(K_{i}\right)=k_{n}$. Consider the fibre square:

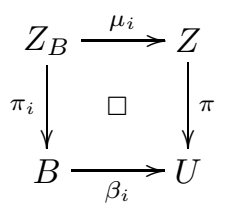

We have the exact sequence

$$
0 \longrightarrow \pi_{i *} \mu_{i}^{*} L^{n} \longrightarrow \beta_{i}^{*} F^{0} \stackrel{\beta_{i}^{*} \varphi}{\longrightarrow} \beta_{i}^{*} F^{1} \longrightarrow R^{1}\left(\pi_{i}\right)_{*} \mu_{i}^{*} L^{n} \longrightarrow 0 .
$$

Note that the quotient sheaf $\beta_{i}^{*} F^{0} / \pi_{i *} \mu_{i}^{*} L^{n}$ is locally free, and $\pi_{i *} \mu_{i}^{*} L^{n}$ and $K_{i}$ are subbundles of $\beta_{i}^{*} F^{0}$ that agree on $B \backslash\{0\}$. It follows that they are isomorphic on $B$. By Proposition [3.3. $\left.\left[\left(\pi_{1}\right)_{*} \mu_{1}^{*} L^{n}\right]\right|_{0}$ and $\left.\left[\left(\pi_{2}\right)_{*} \mu_{2}^{*} L^{n}\right]\right|_{0}$ are identified in $H^{0}\left(\left.Z\right|_{\beta_{i}(0)},\left.L^{n}\right|_{\left.Z\right|_{\beta_{i}(0)}}\right)$. This implies that $\widetilde{\tau} \circ \widetilde{\beta}_{1}(0)=\widetilde{\tau} \circ \widetilde{\beta}_{2}(0)$ and that there exists a subbundle $K$ of $F^{0}$ over $U$ such that $\left.\left.K\right|_{U \backslash Y} \simeq \pi_{*} L^{n}\right|_{U \backslash Y}$. Let $j$ denote the natural inclusion $U \backslash Y \hookrightarrow U$. Since $\pi_{*} L^{n}$ is the kernel of a homomorphism of locally-free sheaves, it is reflexive, and $j_{*}\left(\left.\pi_{*} L^{n}\right|_{U \backslash Y}\right) \simeq \pi_{*} L^{n}$ since $\operatorname{codim}(Y) \geq 2$. Therefore, we have

$$
K \simeq j_{*}\left(\left.K\right|_{U \backslash Y}\right)=j_{*}\left(\left.\pi_{*} L^{n}\right|_{U \backslash Y}\right) \simeq \pi_{*} L^{n},
$$

and hence $\pi_{*} L^{n}$ is a vector subbundle of $F^{0}$. 


\subsection{Analysis of the morphisms.}

Proposition 3.6. For $n \geq 2$, the sections of $L^{n}$ relative to $\pi$ induce a morphism $Z \rightarrow \mathbb{P}\left(\pi_{*} L^{n}\right)$ over $U$, which factors

$$
Z \stackrel{\xi_{Z}}{\rightarrow} \mathcal{T}(Z) \hookrightarrow \mathbb{P}\left(\pi_{*} L^{n}\right),
$$

where the second arrow is a closed embedding.

Proof. By Proposition $3.5 \pi_{*} L^{n}$ is locally free. For $u \in U \backslash W,\left.\pi_{*} L^{n}\right|_{u}=\Gamma\left(\omega_{Z_{u}}^{\otimes n}\right)$ and $\omega_{Z_{u}}^{\otimes n}$ is very ample on stable curves of genus $g>2$. For $u \in W$, Corollary 3.4 implies $\left.\pi_{*} L^{n}\right|_{u}=V_{n}$ induces the morphism replacing elliptic tails with cusps.

We therefore have a morphism $Z \rightarrow \mathbb{P}\left(\pi_{*} L^{n}\right)$ whose Stein factorization is $\mathcal{T}(Z)$. Due to the functoriality of the relative dualizing sheaf and the line bundle $\delta_{1,1}$ on the moduli stack $\overline{\mathcal{M}}_{g, 1}$, this construction is compatible with isomorphism relation and commutes with base extension.

\section{4. $\bar{M}_{g}^{p s}$ AS A LOG CANONiCAL MOdel For $\bar{M}_{g}$}

In this section, we identify $\bar{M}_{g}^{p s}$ and $\bar{M}_{g}(9 / 11)$, thus completing our proof of Theorem 1.1. We also prove Theorem 1.2. A log canonical model for the pair $\left(\bar{M}_{g}, K_{\overline{\mathcal{M}}_{g}}+\alpha \delta\right)$ exists as a projective variety for $7 / 10<\alpha \leq 9 / 11$.

We want to relate the contraction of the extremal ray $R$ parametrizing elliptic tails

$$
\Upsilon: \bar{M}_{g} \rightarrow \bar{M}_{g}(9 / 11)
$$

to the natural birational morphism

$$
T: \bar{M}_{g} \rightarrow \bar{M}_{g}^{p s}
$$

Since $\bar{M}_{g}^{p s}$ is a GIT quotient, it is a normal projective variety. Our pointwise description of the natural transformation $\mathcal{T}: \overline{\mathcal{M}}_{g} \rightarrow \overline{\mathcal{M}}_{g}^{p s}$ in Proposition 3.1 implies that

- $T$ is an isomorphism over $\bar{M}_{g} \backslash \Delta_{1}$;

- $T$ takes $R\left(C_{2}, p\right)$ to the cuspidal curve $\mathcal{T}\left(C_{1} \cup_{p} C_{2}\right)$, i.e., the curve normalized by $C_{2}$ with conductor in $p$;

- $\mathcal{T}(C)=\mathcal{T}\left(C^{\prime}\right)$ if and only if $C$ and $C^{\prime}$ have the same number of elliptic tails and

$$
\left(D, p_{1}, \ldots, p_{r}\right) \simeq\left(D^{\prime}, p_{1}^{\prime}, \ldots, p_{r}^{\prime}\right)
$$

in the notation of Proposition 3.1

In particular, the only curves contracted by $T$ are in the class $R$, i.e., $T$ is also an extremal contraction of $R$. Uniqueness of extremal contractions KM98, 1.26 implies $T=\Upsilon$ and $\bar{M}_{g}^{p s} \simeq \bar{M}_{g}(9 / 11)$, and the proof of Theorem 1.1 is complete.

We turn to the proof of Theorem [1.2, Let $\delta^{p s}$ denote the boundary divisor of $\overline{\mathcal{M}}_{g}^{p s}$; it is the image of $\delta$ under $\mathcal{T}$. The proper transform of $\delta^{p s}$ in $\overline{\mathcal{M}}_{g}$ is $\delta-\delta_{1}$.

Lemma 4.1 (Log discrepancy formula).

$$
K_{\overline{\mathcal{M}}_{g}}+\alpha \delta=T^{*}\left(K_{\overline{\mathcal{M}}_{g}^{p s}}+\alpha \delta^{p s}\right)+(9-11 \alpha) \delta_{1} .
$$


Proof. We determine the value of the discrepancy $c$ in

$$
K_{\overline{\mathcal{M}}_{g}}+\alpha \delta=T^{*}\left(K_{\overline{\mathcal{M}}_{g}^{p s}}+\alpha \delta^{p s}\right)+c \delta_{1}
$$

by intersecting both sides with the contracted extremal ray $R$. We have

$$
\begin{aligned}
\left(K_{\overline{\mathcal{M}}_{g}}+\alpha \delta\right) \cdot R & =(13 \lambda-2 \delta+\alpha \delta) \cdot R=13 \lambda \cdot R-(2-\alpha)\left(\delta_{0} \cdot R+\delta_{1} \cdot R\right) \\
& =13 / 12-(2-\alpha)(1-1 / 12)=(11 \alpha-9) / 12
\end{aligned}
$$

and $c \delta_{1} \cdot R=-c / 12$; the term $T^{*}\left(K_{\overline{\mathcal{M}}_{g}^{p s}}+\alpha \delta^{p s}\right)$ obviously yields zero.

Thus $\left(\overline{\mathcal{M}}_{g}^{p s}, \alpha \delta^{p s}\right)$ has $\log$ canonical singularities for $\alpha \leq 9 / 11$ provided $\left(\overline{\mathcal{M}}_{g}, \alpha \delta-(9-11 \alpha) \delta_{1}\right)$ has log canonical singularities. The argument sketched in the Introduction still applies away from $\Delta_{1}$. However, reducing the coefficient of $\delta_{1}$ can only increase the discrepancies of divisors lying over $\delta_{1}$ GK92], 2.17.3; this does not affect whether the singularities are log canonical.

To analyze whether $K_{\overline{\mathcal{M}}_{g}^{p s}}+\alpha \delta^{p s}$ is ample for $7 / 10<\alpha<9 / 11$, we describe the ample cone of $\bar{M}_{g}^{p s}$ in terms of the ample cone of $\bar{M}_{g}$. We have

$$
T^{*} \mathrm{NS}\left(\bar{M}_{g}^{p s}\right)=R^{\perp} \subset \mathrm{NS}\left(\bar{M}_{g}\right),
$$

the hyperplane spanned by a facet of the nef cone of $\bar{M}_{g}$. The interior of this facet corresponds to the ample divisors of $\bar{M}_{g}^{p s}$. It suffices then to check:

Proposition 4.2. For $7 / 10<\alpha \leq 9 / 11$, the divisor

$$
K_{\overline{\mathcal{M}}_{g}}+\alpha \delta-(9-11 \alpha) \delta_{1}
$$

lies in the interior of the facet $R^{\perp}$ of the nef cone of $\bar{M}_{g}$.

The following conjectural description of the ample cone of $\bar{M}_{g, n}$ would end the discussion in one stroke:

Conjecture 4.3 (Fulton's conjecture GKM02]). Every one-dimensional facet of the closed cone of effective curves $\overline{N E}_{1}\left(\bar{M}_{g, n}\right)$ is generated by a one-dimensional boundary stratum. Equivalently, any effective curve in $\bar{M}_{g, n}$ is an effective combination of one-dimensional strata.

We recapitulate the description of the one-dimensional strata in [Fab96] and GKM02: Let $X_{0}$ be a 4-pointed stable curve of genus zero with one point moving and the other three fixed.

(a) a family of elliptic tails;

(b) attach a fixed 4-pointed curve of genus $g-3$ to $X_{0}$;

(c) attach a fixed pointed curve of genus $i$ and a 3-pointed curve of genus $g-2-i$ to $X_{0}$, with $g-2 \geq i \geq 0$

(d) attach two fixed 2-pointed curves of genus $i$ and $g-2-i$, respectively, to $X_{0}$, with $(g-2) / 2 \geq i \geq 0$

(e) attach two 1-pointed curves of genus $i$ and $j$, respectively, and a 2-pointed curve of genus $g-1-i-j$ to $X_{0}$, with $i, j \geq 1$ and $i+j \leq g-1$;

(f) attach four pointed curves of genus $i, j, k$, and $l$, respectively, to $X_{0}$, with $i+j+k+l=g$ and $i, j, k \geq 1$.

Where we attach a 2-pointed curve of genus zero, we stabilize the resulting 'rational bridge' to a node. 
The intersection of a divisor $a \lambda-\sum_{i=0}^{\lfloor g / 2\rfloor} b_{i} \delta_{i}$ with the one-dimensional strata is as follows:

\begin{tabular}{|c|c|}
\hline (a) & $a / 12-b_{0}+b_{1} / 12$ \\
\hline (b) & $b_{0}$ \\
\hline (c) & $b_{i}$ \\
\hline (d) & $2 b_{0}-b_{i+1}$ \\
\hline (e) & $b_{i}+b_{j}-b_{i+j}$ \\
\hline (f) & $b_{i}+b_{j}+b_{k}+b_{l}-b_{i+j}-b_{i+k}-b_{i+l}$ \\
\hline
\end{tabular}

Here we use the convention that $b_{i}=b_{g-i}$ for $i>g / 2$.

Fortunately, we do not need the full strength of the conjecture. The following special case is sufficient:

Proposition 4.4 (GKM02, 6.1]). Let $D=a \lambda-\sum_{0 \leq i \leq g / 2} b_{i} \delta_{i}$ be a divisor on $\bar{M}_{g}$ such that either $b_{i}=0$ or $b_{i} \geq b_{0}, 1 \leq i \leq\lfloor g / 2\rfloor$. If D has non-negative intersection with all the one-dimensional strata, then $D$ is nef.

In our case, we have

$$
a=13, \quad b_{i}=\left\{\begin{array}{l}
2-\alpha, \quad i \neq 1, \\
11-12 \alpha, \quad i=1,
\end{array}\right.
$$

and our divisor satisfies the hypothesis of Proposition 4.4 as long as $\alpha \leq 9 / 11$. We consider the following when each class of one-dimensional stratum intersects our divisor positively:

- For (b), (c) and (f), we have positivity provided $\alpha \leq 11 / 12$.

- For (d), we have positivity provided $2(2-\alpha)-(11-12 \alpha)>0$, i.e., $\alpha>7 / 10$; the case $i=0$ shows this is sharp.

- For (e), we have positivity provided $\alpha>7 / 10$; the case $i+j=g-1, i \neq$ $1, j \neq 1$ shows this is sharp.

Proposition 4.2 therefore follows from Proposition 4.4. This completes the proof of Theorem 1.2 ,

Remark 4.5. $T^{*}\left(K_{\overline{\mathcal{M}}_{g}^{p s}}+7 / 10 \delta^{p s}\right)$ contracts $\Delta_{1}$ and the following loci:

- the stratum (d) with $i=0$ induces the contraction of

$$
T_{0}=\left\{C_{1} \cup_{p, q} C_{2} \mid g\left(C_{1}\right)=1, g\left(C_{2}\right)=g-2\right\},
$$

the locus of two-pointed elliptic tails or elliptic bridges;

- the stratum (e) with $i+j=g-1$ and $i, j \neq 1$ induces the contraction of

$T_{i}=\left\{C_{1} \cup_{p} C_{2} \cup_{q} C_{3} \mid g\left(C_{1}\right)=i, g\left(C_{2}\right)=1, g\left(C_{3}\right)=g-1-i\right\}, \quad i=2, \ldots,\lfloor(g-1) / 2\rfloor$.

These have codimension two in the moduli space; at $\alpha=7 / 10$ we obtain a small contraction.

Remark 4.6. For a special value of $\alpha \in(7 / 10,9 / 11)$, we can prove the ampleness of $K_{\overline{\mathcal{M}}_{g}^{p s}}+\alpha \delta^{p s}$ using invariant theory. Recall from $\S 2$ that

$$
\bar{M}_{g}^{p s} \simeq \mathrm{Chow}_{3} / / \mathrm{SL}_{5(g-1)},
$$

where the Chow variety has its natural linearization. By Theorem 5.15 of Mum77, the corresponding line bundle on $\bar{M}_{g}$ equals $3(g-1)(3(12 \lambda-\delta)-4 \lambda)$, which is proportional to $32 \lambda-3 \delta \sim K_{\overline{\mathcal{M}}_{g}}+25 / 32 \delta$. This is not ample on $\bar{M}_{g}$ since $\Delta_{1}$ is a 
fixed component. However, the corresponding divisor $K_{\overline{\mathcal{M}}_{g}^{p s}}+25 / 32 \delta^{p s}$ on $\bar{M}_{g}^{p s}$ is ample.

\section{Appendix A. Log CANONICAL MOdELS OF STACKS}

Here are conventions for this section: A 'scheme' is a separated scheme of finite type over $k$. A 'stack' is a Deligne-Mumford stack, separated and of finite type over $k$; a 'morphism' is a 1-morphism over the base field. For a general discussion of local properties of representable morphisms, we refer the reader to [LMB00, 3.10] and Gro65, 2.6,2.7]; we use these without specific attribution. Roughly, every property that behaves well under étale basechange makes sense for Deligne-Mumford stacks.

Definition A.1. A birational morphism of stacks is a morphism $f: \mathcal{X}_{1} \rightarrow \mathcal{X}_{2}$ such that there exist dense open substacks $\mathcal{U}_{1} \subset \mathcal{X}_{1}$ and $\mathcal{U}_{2} \subset \mathcal{X}_{2}$ with $\mathcal{U}_{2}=f^{-1}\left(\mathcal{U}_{1}\right)$ and $f: \mathcal{U}_{1} \rightarrow \mathcal{U}_{2}$ an isomorphism. We say that $\mathcal{X}_{1}$ and $\mathcal{X}_{2}$ are properly birational if there exists a stack $\mathcal{Y}$ and birational proper morphisms $g_{1}: \mathcal{Y} \rightarrow \mathcal{X}_{1}$ and $g_{2}: \mathcal{Y} \rightarrow \mathcal{X}_{2}$.

It is straightforward to check that this is an equivalence relation. There is a distinguished open substack $\mathcal{U} \subset \mathcal{X}_{1}$ which is the largest open set over which $f$ is an isomorphism; its complement is called the exceptional locus $\operatorname{Ex}(f)$. For any closed substack $\mathcal{D} \subset \mathcal{X}_{2}$ with $f(\mathcal{U}) \cap \mathcal{D}$ dense in $\mathcal{D}$, the birational transform $f_{*}^{-1} \mathcal{D}$ is defined as the closure of $f^{-1}(\mathcal{D} \cap f(\mathcal{U}))$ in $\mathcal{X}_{1}$.

Proposition A.2. For each reduced stack $\mathcal{X}$, there exists a smooth stack $\tilde{\mathcal{X}}$ and a birational proper morphism $f: \tilde{\mathcal{X}} \rightarrow \mathcal{X}$; this is called a resolution of $\mathcal{X}$. Furthermore, $\operatorname{Ex}(f)$ can be taken to be a normal crossings divisor in $\tilde{X}$. If $\mathcal{Z} \hookrightarrow \mathcal{X}$ is a closed substack with ideal sheaf $I_{\mathcal{Z}} \subset \mathcal{O}_{\mathcal{X}}$, then $f$ can be chosen so that $f^{*} I_{\mathcal{Z}}$ is an invertible sheaf $\mathcal{O}_{\tilde{\mathcal{X}}}(-\widetilde{\mathcal{D}})$ and $\operatorname{Ex}(f) \cup \widetilde{\mathcal{D}}$ is simple normal crossings. This is called $a \log$ resolution of $\mathcal{Z}$ in $\mathcal{X}$.

Proof. This statement should be compared to Theorem 0.2 of KM98. It is an application of the functorial procedure for resolving singularities [BM97]: Such procedures commute with étale base extension [Hau03], pp. 329. In particular, given an étale presentation of $\mathcal{X}$

$$
R \rightrightarrows U
$$

canonical resolutions $\widetilde{R} \rightarrow R$ and $\widetilde{U} \rightarrow U$ form a presentation

$$
\widetilde{R} \rightrightarrows \widetilde{U}
$$

for a smooth stack $\widetilde{\mathcal{X}}$. The induced representable morphism $\widetilde{\mathcal{X}} \rightarrow \mathcal{X}$ is birational and proper. If $E \subset U$ denotes the closed subscheme corresponding to $\mathcal{Z}$, then the canonical embedded resolution of $E$ in $U$ naturally induces a resolution of $\mathcal{Z}$ in $\mathcal{X}$.

Remark A.3. Dan Abramovich has pointed out that the 1992 Harvard thesis of Andrew Joel Schwartz, Functorial Smoothing Of Morphisms In Equal Characteristic 0 , should also suffice for our purposes.

Let $X$ be a normal connected scheme, separated and of finite type over a field, as usual. Let $D=\sum a_{j} D_{j}$ be a $\mathbb{Q}$-divisor on $X$, with the $D_{j} \subset X$ distinct codimension-one reduced closed subschemes; we assume each $a_{j} \in[0,1]$. 
Proposition A.4. The following properties of $(X, D)$ are local in the étale topology on $X$ :

(1) $X$ is normal;

(2) $D_{j}$ is a codimension-one reduced closed subscheme;

(3) $m\left(K_{X}+D\right)$ is Cartier for some $m>0$.

A pair $(X, D)$ satisfying the first three conditions is said to be admissible. Given a coherent $\mathcal{O}_{X}$-module $F$, the following properties are local in étale topology:

(1) F satisfies Serre's condition $S_{k}$ for some fixed $k>0$;

(2) $F$ is locally free.

Proof. These are standard properties of descent: See Gro65, §6.1, 6.4, 6.5, and 6.8 for the behavior of dimension, property $S_{k}$, property $R_{k}$, and other standard singularity conditions under faithfully flat basechange. See Gro65], 2.5.1 for the stability of local-freeness under faithfully flat base extension.

Proposition A.5. Let $X$ be a normal integral scheme with field of fractions $K(X)$, and $D$ be an integral Weil divisor on $X$. Let $\mathcal{O}_{X}(D)$ denote the $\mathcal{O}_{X}$-module associated to

$$
\{f \in K(X): \operatorname{div}(f)+D \geq 0\} .
$$

This is a coherent $\mathcal{O}_{X}$-module of rank one, satisfying Serre's condition $S_{2}$. The formation of $\mathcal{O}_{X}(D)$ commutes with étale base-extension.

Proof. Let $j: U \subset X$ be the locus where $X$ is smooth, which is compatible with étale basechange. Then $\mathcal{O}_{X}(D)=j_{*} \mathcal{O}_{U}(D \cap U)$, which is coherent, $S_{2}$, and compatible with base extension.

Let $\mathcal{X}$ denote a normal connected stack. Let $\mathcal{D}=\sum a_{j} \mathcal{D}_{j}$ be a $\mathbb{Q}$-divisor on $\mathcal{X}$, where the $\mathcal{D}_{j} \subset \mathcal{X}$ are distinct codimension-one reduced closed substacks; we assume each $a_{j} \in[0,1]$.

Definition A.6. Suppose $(\mathcal{X}, \mathcal{D})$ is proper and admissible in the sense of Proposition A.4. Its log canonical ring is the graded ring

$$
R(\mathcal{X}, \mathcal{D}):=\bigoplus_{m \geq 0} \Gamma\left(\mathcal{X}, \mathcal{O}_{\mathcal{X}}\left(m K_{\mathcal{X}}+\lfloor m \mathcal{D}\rfloor\right)\right)
$$

where

$$
\lfloor m \mathcal{D}\rfloor=\sum_{j}\left\lfloor m a_{j}\right\rfloor \mathcal{D}_{j},
$$

and the summands are defined via Proposition A.5.

We refer the reader to $\S 2.3$ of KM98 for definitions of the various singularities arising in birational geometry.

Proposition A.7 (Proposition 5.20 of KM98). Let $(X, D)$ be an admissible pair, as in Proposition A.4, defined over a field of characteristic zero. The following singularity conditions are local in the étale topology: terminal, canonical, Kawamata log terminal, or log canonical.

Hence the following notions are well-defined:

Definition A.8. $(\mathcal{X}, \mathcal{D})$ is terminal (resp. canonical, Kawamata log terminal, or $\log$ canonical) if it admits an étale presentation with the same property. It is strictly $\log$ canonical if it is $\log$ canonical and $\mathcal{X} \backslash \bigcup_{i} \mathcal{D}_{i}$ is canonical. 
The reader will recall that these notions are defined in terms of discrepancies KM98, §2.3, measuring how the canonical divisor changes under birational morphisms:

Proposition A.9. The admissible pair $(\mathcal{X}, \mathcal{D})$ is terminal (resp. canonical, Kawamata log terminal, or log canonical) if and only if there exists a log resolution $f: \widetilde{\mathcal{X}} \rightarrow \mathcal{X}$ such that

(1) $\operatorname{Ex}(f)=\bigcup \mathcal{E}_{i}$ is a divisor, $\operatorname{Ex}(f) \cup f^{-1}(\mathcal{D})$ is normal crossings, and $\sum_{j} f_{*}^{-1} \mathcal{D}_{j}$ is smooth;

(2) we have a $\mathbb{Q}$-linear equivalence

$(*) \quad K_{\tilde{\mathcal{X}}}+\sum_{j} a_{j} f_{*}^{-1} \mathcal{D}_{j} \equiv f^{*}\left(K_{\mathcal{X}}+\mathcal{D}\right)+\sum_{i} d\left(\mathcal{E}_{i} ; \mathcal{X}, \mathcal{D}\right) \mathcal{E}_{i}, \quad d_{i}:=d\left(\mathcal{E}_{i} ; \mathcal{X}, \mathcal{D}\right)$,

with each $a_{j}<1$ and $d_{i}>0$ (resp. $a_{j} \leq 1$ and $d_{i} \geq 0, a_{j}<1$ and $d_{i}>-1$, or $a_{j} \leq 1$ and $d_{i} \geq-1$ ).

Proof. Proposition A.2 guarantees there exists a resolution with the prescribed properties. By definition, if $(\mathcal{X}, \mathcal{D})$ has the prescribed singularities, then the discrepancies $d_{i}$ and coefficients $a_{j}$ satisfy the listed properties. The converse direction is an application of Corollary 2.32 of [KM98].

Definition A.10. The discrepancy $d\left(\mathcal{E}_{0} ; \mathcal{X}, \mathcal{D}\right)$ is defined via equation $(*)$ for any integral codimension-one substack in the exceptional locus of some birational proper morphism $\mathcal{Y} \rightarrow \mathcal{X}$. By convention, we set

$$
d_{j}=d\left(\mathcal{D}_{j} ; \mathcal{X}, \mathcal{D}\right)=-a_{j}
$$

and take $d\left(\mathcal{D}_{0} ; \mathcal{X}, \mathcal{D}\right)=0$ for any integral codimension-one substack $\mathcal{D}_{0} \subset \mathcal{X}$ not in the support of $\mathcal{D}$.

Definition A.11. Two admissible pairs $(\mathcal{X}, \mathcal{D})$ and $\left(\mathcal{X}^{\prime}, \mathcal{D}^{\prime}\right)$ are properly birational if there exists an admissible pair $(\widetilde{\mathcal{X}}, \widetilde{\mathcal{D}})$ and proper birational morphisms $f: \widetilde{\mathcal{X}} \rightarrow$ $\mathcal{X}, f^{\prime}: \widetilde{\mathcal{X}} \rightarrow \mathcal{X}^{\prime}$, so that $K_{\tilde{\mathcal{X}}}+\widetilde{\mathcal{D}}-f^{*}\left(K_{\mathcal{X}}+\mathcal{D}\right)\left(\operatorname{resp} . K_{\tilde{\mathcal{X}}}+\widetilde{\mathcal{D}}-f^{\prime *}\left(K_{\mathcal{X}^{\prime}}+\mathcal{D}^{\prime}\right)\right)$ is effective and $f$-exceptional (resp. $f^{\prime}$-exceptional).

Our main interest is the case where $(\mathcal{X}, \mathcal{D})$ and $\left(\mathcal{X}^{\prime}, \mathcal{D}^{\prime}\right)$ are log canonical and $(\widetilde{\mathcal{X}}, \widetilde{\mathcal{D}})$ is a $\log$ resolution with suitably chosen boundary.

Proposition A.12. If the admissible pairs $(\mathcal{X}, \mathcal{D})$ and $\left(\mathcal{X}^{\prime}, \mathcal{D}^{\prime}\right)$ are properly birational, then there is a natural isomorphism

$$
R(\mathcal{X}, \mathcal{D}) \stackrel{\sim}{\rightarrow} R\left(\mathcal{X}^{\prime}, \mathcal{D}^{\prime}\right)
$$

of graded rings.

Proof. It suffices to prove the isomorphism

$$
R(\mathcal{X}, \mathcal{D}) \stackrel{\sim}{\rightarrow} R(\widetilde{\mathcal{X}}, \widetilde{\mathcal{D}})
$$

We have the discrepancy equation

$$
K_{\tilde{\mathcal{X}}}+\widetilde{\mathcal{D}}={ }_{\mathbb{Q}} f^{*}\left(K_{\mathcal{X}}+\mathcal{D}\right)+\sum_{i} d_{i} \mathcal{E}_{i}
$$


where the $d_{i} \geq 0$ and the $\mathcal{E}_{i}$ are $f$-exceptional. This was formulated on the level of $\mathbb{Q}$-Cartier divisors, but there is a refined interpretation: For each $m \geq 0$, consider the homomorphisms

$(* *) \quad f^{*} \mathcal{O}_{\mathcal{X}}\left(m K_{\mathcal{X}}+\lfloor m \mathcal{D}\rfloor\right) \rightarrow \mathcal{O}_{\tilde{\mathcal{X}}}\left(m K_{\tilde{\mathcal{X}}}+\left\lfloor m\left(\widetilde{\mathcal{D}}-\sum_{i} d_{i} \mathcal{E}_{i}\right)\right\rfloor\right) \subset \mathcal{O}_{\tilde{\mathcal{X}}}\left(m K_{\tilde{\mathcal{X}}}+\lfloor m \widetilde{\mathcal{D}}\rfloor\right)$.

These deserve some explanations: First, there is no harm passing to an étale neighborhood which is a scheme; for simplicity, we leave the notation unchanged. The divisors $\left\lfloor m f_{*}^{-1} \mathcal{D}\right\rfloor$ and $\left\lfloor m\left(\widetilde{\mathcal{X}}-\sum_{i} d_{i} \mathcal{E}_{i}\right)\right\rfloor$ agree on all the components which have codimension one in $\mathcal{X}$, so we can focus on the $f$-exceptional components. Every section $s \in \mathcal{O}_{\mathcal{X}}\left(m K_{\mathcal{X}}+\lfloor m \mathcal{D}\rfloor\right)$ extends to a section with poles of some order on the exceptional locus of $f$. Replacing $s$ by a suitable power $s^{N}$, we may assume that $m N\left(K_{\mathcal{X}}+\mathcal{D}\right)$ is integral and Cartier, and our discrepancy equation gives the requisite bound on the pole order. The inclusions $(* *)$ induce injections

$$
\Gamma\left(m K_{\mathcal{X}}+\lfloor m \mathcal{D}\rfloor\right) \hookrightarrow \Gamma\left(m K_{\tilde{\mathcal{X}}}+\lfloor m \widetilde{\mathcal{D}}\rfloor\right) .
$$

This is surjective: Each element in the target of the map is a section of

$$
\Gamma\left(\mathcal{X}-\bigcup_{i} f\left(\mathcal{E}_{i}\right), m K_{\mathcal{X}}+\lfloor m \mathcal{D}\rfloor\right),
$$

which extends to all of $\mathcal{X}$ because $\mathcal{X}$ is normal.

Proposition A.13. Let $(\mathcal{X}, \mathcal{D})$ be a proper log canonical (resp. Kawamata log terminal) pair, and let $\pi: \mathcal{X} \rightarrow X$ denote the coarse moduli space of $X$. Then there exists an effective $\mathbb{Q}$-divisor $\check{D}=\sum_{\ell} \check{c}_{\ell} \check{D}_{\ell}, 0 \leq \check{c}_{\ell} \leq 1$ on $X$, with the $\check{D}_{\ell}$ distinct and irreducible with the following properties:

(1) $(X, \check{D})$ is log canonical (resp. Kawamata log terminal);

(2) for each positive integer $m$ such that $m\left(K_{X}+\check{D}\right)$ is integral and Cartier, there is an equivalence of Cartier divisors

$$
m\left(K_{\mathcal{X}}+\mathcal{D}\right)=\pi^{*} m\left(K_{X}+\check{D}\right)
$$

(3) for every $m \geq 0$ we have

$$
\Gamma\left(X, m K_{X}+\lfloor m \check{D}\rfloor\right) \stackrel{\sim}{\rightarrow} \Gamma\left(\mathcal{X}, m K_{\mathcal{X}}+\lfloor m \mathcal{D}\rfloor\right) .
$$

Together, these yield a natural isomorphism

$$
\pi^{*}: R(X, \check{D}) \stackrel{\sim}{\rightarrow} R(\mathcal{X}, \mathcal{D}) .
$$

Proof. We first produce the divisor $\check{D}$; to extract its coefficients, we localize along each codimension-one integral closed substack $\mathcal{B} \subset \mathcal{X}$. Let $a(\mathcal{B})$ denote the coefficient of $\mathcal{D}$ at $\mathcal{B}$, which might be zero if $\mathcal{B}$ does not appear in $\mathcal{D}$. Suppose $\pi$ ramifies to order $e(\mathcal{B})$ at the generic point $\eta(\mathcal{B})$; i.e., the order of the automorphism group at the generic point of $\mathcal{B}$ is $e(\mathcal{B})$ times the order of the automorphism group at the generic point of $\mathcal{X}$. If $B \subset X$ is the reduced image of $\mathcal{B}$, then the ramification formula gives

$$
\pi^{*} \omega_{X, \eta(B)}=\omega_{\mathcal{X}, \eta(\mathcal{B})}(-(e(\mathcal{B})-1) \mathcal{B}), \quad \pi^{*} B=e(\mathcal{B}) \mathcal{B}
$$

which yields the equation of $\mathbb{Q}$-Cartier divisors

$$
\pi^{*}\left(K_{X}+\sum_{\mathcal{B}} \frac{e(\mathcal{B})-1+a(\mathcal{B})}{e(\mathcal{B})} B\right) \equiv K_{\mathcal{X}}+\mathcal{D}
$$


We therefore set

$$
\check{D}=\sum_{\mathcal{B}} \frac{e(\mathcal{B})-1+a(\mathcal{B})}{e(\mathcal{B})} B
$$

the pair $(X, \check{D})$ is log canonical (resp. Kawamata log terminal) by [KM98, 5.20.

It then remains to check

$$
\Gamma\left(X, m K_{X}+\sum_{B}\left\lfloor m \frac{e(\mathcal{B})-1+a(\mathcal{B})}{e(\mathcal{B})}\right\rfloor B\right) \simeq \Gamma\left(\mathcal{X}, m K_{\mathcal{X}}+\sum_{\mathcal{B}}\lfloor m a(\mathcal{B})\rfloor \mathcal{B}\right) .
$$

By the ramification formulas, we have

$$
\begin{array}{r}
\Gamma\left(X, m K_{X}+\sum_{B}\left\lfloor m \frac{e(\mathcal{B})-1+a(\mathcal{B})}{e(\mathcal{B})}\right\rfloor B\right) \\
\hookrightarrow \Gamma\left(\mathcal{X}, m K_{\mathcal{X}}+\sum_{\mathcal{B}}\left(e(\mathcal{B})\left\lfloor m \frac{e(\mathcal{B})-1+a(\mathcal{B})}{e(\mathcal{B})}\right\rfloor-m(e-1)\right) \mathcal{B}\right) .
\end{array}
$$

This is surjective: For any $\mathcal{O}_{X}$-module $F$ we have $\Gamma(X, F)=\Gamma\left(\mathcal{X}, \pi^{*} \mathcal{F}\right)$, as every section of $\pi^{*} \mathcal{F}$ is pulled back from the coarse moduli space. We must verify that the inclusion

$$
\begin{array}{r}
\Gamma\left(\mathcal{X}, m K_{\mathcal{X}}+\sum_{\mathcal{B}}\left(e(\mathcal{B})\left\lfloor m \frac{e(\mathcal{B})-1+a(\mathcal{B})}{e(\mathcal{B})}\right\rfloor-m(e(\mathcal{B})-1)\right) \mathcal{B}\right) \\
\subset \Gamma\left(\mathcal{X}, m K_{\mathcal{X}}+\sum_{\mathcal{B}}\lfloor m a(\mathcal{B})\rfloor \mathcal{B}\right)
\end{array}
$$

is an isomorphism. We analyze sections locally in an étale neighborhood of $\eta(\mathcal{B})$, where the stack can be presented as a $\mu_{e}$-quotient, with $e=e(\mathcal{B})$ (Lemma 2.2.3 of AV02); fix $a=a(\mathcal{B})$. Let $\omega$ be a local generator of the canonical class and $F$ the local defining equation for $\mathcal{B}$, so that $\mu_{e}$ acts on both $\omega$ and $F$ via multiplication by $\zeta$, a primitive $e$ th root of unity. The sheaf $\mathcal{O}_{\mathcal{X}}\left(m K_{\mathcal{X}}+\sum_{\mathcal{B}}\lfloor m a(\mathcal{B})\rfloor \mathcal{B}\right)$ has a local generator $\omega^{\otimes m} / F^{\lfloor m a\rfloor}$, on which $\mu_{e}$ acts by $\zeta^{m-\lfloor m a\rfloor}$. Of course, $\mu_{e}$ acts trivially on global sections, so global sections must be multiples of

$$
F^{\lfloor m a\rfloor-m-e\lfloor(\lfloor m a\rfloor-m) / e\rfloor} \omega^{\otimes m} / F^{\lfloor m a\rfloor}=\omega^{\otimes m} / F^{m+e\lfloor(\lfloor m a\rfloor-m) / e\rfloor} .
$$

Thus global sections of $\mathcal{O}_{\mathcal{X}}\left(m K_{\mathcal{X}}+\sum_{\mathcal{B}}\lfloor m a(\mathcal{B})\rfloor \mathcal{B}\right)$ are forced to have zeros along $\mathcal{B}$; precisely, we find

$$
\Gamma\left(m K_{\mathcal{X}}+\sum_{\mathcal{B}}\left(m+e(\mathcal{B})\left\lfloor\frac{\lfloor m a(\mathcal{B})\rfloor-m}{e(\mathcal{B})}\right\rfloor\right) \mathcal{B}\right)=\Gamma\left(m K_{\mathcal{X}}+\sum_{\mathcal{B}}\lfloor m a(\mathcal{B})\rfloor \mathcal{B}\right) .
$$

The final step is the combinatorial statement

$$
e\left\lfloor m \frac{e-1+a}{e}\right\rfloor-m(e-1)=m+e\lfloor(\lfloor m a\rfloor-m) / e\rfloor,
$$

which is equivalent to

$$
\left\lfloor\frac{m a-m}{e}\right\rfloor=\lfloor(\lfloor m a\rfloor-m) / e\rfloor .
$$

Here $e$ and $m$ are positive integers and $a \in[0,1] \cap \mathbb{Q}$. If this were false, then we could find an integer $n$ with

$$
(\lfloor m a\rfloor-m) / e<n \leq(m a-m) / e
$$


and hence

$$
\lfloor m a\rfloor<e n+m \leq m a,
$$

violating the definition of the round-down operation.

The Kawamata basepoint-freeness theorem KM98 yields

Corollary A.14 (Basepoint-freeness for stacks). Let $(\mathcal{X}, \mathcal{D})$ be a proper Kawamata log terminal pair. Assume $K_{\mathcal{X}}+\mathcal{D}$ is nef and big, i.e., $K_{X}+\check{D}$ is nef and big, where $(X, \check{D})$ is the pair constructed in Proposition A.13. Consider the quotient stack and coarse moduli space

$$
\mathcal{Y}:=\left[(\operatorname{Spec} R(\mathcal{X}, \mathcal{D}) \backslash 0) / \mathbb{G}_{m}\right] \quad Y:=\operatorname{Proj} R(\mathcal{X}, \mathcal{D})=\operatorname{Proj} R(X, \check{D}),
$$

where the action of $\mathbb{G}_{m}$ arises from the grading. Then there is a morphism of stacks

$$
\psi: \mathcal{X} \rightarrow \mathcal{Y}
$$

inducing on coarse moduli spaces the contraction from $X$ to the log canonical model of $(X, \check{D})$.

\section{ACKNOWLEDGMENTS}

The first author was partially supported by National Science Foundation grants 0196187 and 0134259, the Sloan Foundation, and the Institute of Mathematical Sciences of the Chinese University of Hong Kong. The second author was partially supported by the Korea Institute for Advanced Study and a Korea Research Foundation Grant funded by the Korean Government (MOEHRD) (KRF-2005-042C00005). We owe a great deal to S. Keel, who helped shape our understanding of the birational geometry of $\bar{M}_{g}$ through detailed correspondence. We gratefully acknowledge Ian Morrison who pointed out Remark 2.4 and an error in an earlier version. We also would like to thank D. Abramovich, Y. Kawamata, B.P. Purnaprajna, and D. Smyth for useful conversations.

\section{REFERENCES}

[AV02] Dan Abramovich and Angelo Vistoli. Compactifying the space of stable maps. J. Amer. Math. Soc., 15(1):27-75 (electronic), 2002. MR.1862797 (2002i:14030)

[BCHM06] Caucher Birkar, Paolo Cascini, Christopher D. Hacon, and James $\mathrm{M}^{\mathrm{c}}$ Kernan. Existence of minimal models for varieties of log general type, 2006. arXiv.org:math/0610203.

[BM97] Edward Bierstone and Pierre D. Milman. Canonical desingularization in characteristic zero by blowing up the maximum strata of a local invariant. Invent. Math., 128(2):207302, 1997. MR1440306 (98e:14010)

[CH88] Maurizio Cornalba and Joe Harris. Divisor classes associated to families of stable varieties, with applications to the moduli space of curves. Ann. Sci. École Norm. Sup. (4), 21(3):455-475, 1988. MR974412 (89j:14019)

[CF91] Fernando Cukierman and Lung-Ying Fong. On higher Weierstrass points. Duke Math. J., 62(1):179-203, 1991. MR.1104328 (92c:14027)

[DM69] Pierre Deligne and David Mumford. The irreducibility of the space of curves of given genus. Inst. Hautes Études Sci. Publ. Math., (36):75-109, 1969. MR 0262240|(41:6850)

[Fab96] Carel Faber. Intersection-theoretical computations on $\bar{M}_{g}$. In Parameter spaces (Warsaw, 1994), volume 36 of Banach Center Publ., pages 71-81. Polish Acad. Sci., Warsaw, 1996. MR 1481481 (98j:14033)

[Far06] Gavril Farkas. The global geometry of the moduli space of curves, 2006. math.AG/0612251.

[GKM02] Angela Gibney, Sean Keel, and Ian Morrison. Towards the ample cone of $\bar{M}_{g, n} . J$. Amer. Math. Soc., 15(2):273-294 (electronic), 2002. MR.1887636 (2003c:14029) 
[GK92] Antonella Grassi and János Kollár. Log canonical models. In János Kollár, editor, Flips and abundance for algebraic threefolds, volume 211 of Astérisque, pages 29-45. Société Mathématique de France, Paris, 1992.

[Gro65] Alexander Grothendieck. Éléments de géométrie algébrique. IV. Étude locale des schémas et des morphismes de schémas. II. Inst. Hautes Études Sci. Publ. Math., (24):231, 1965. MR0199181 (33:7330)

[HKT06] Paul Hacking, Sean Keel, and Jenia Tevelev. Compactification of the moduli space of hyperplane arrangements. J. Algebraic Geom., 15(4):657-680, 2006. MR2237265 (2007j:14016)

[HM82] Joe Harris and David Mumford. On the Kodaira dimension of the moduli space of curves. Invent. Math., 67(1):23-88, 1982. With an appendix by William Fulton. MR664324 (83i:14018)

[Has03] Brendan Hassett. Moduli spaces of weighted pointed stable curves. Adv. Math., 173(2):316-352, 2003. MR.1957831 (2004b:14040)

[Has05] Brendan Hassett. Classical and minimal models of the moduli space of curves of genus two. In Geometric methods in algebra and number theory, volume 235 of Progress in mathematics, pages 160-192. Birkhäuser, Boston, 2005. MR:2166084 (2006g:14047)

[HH08] Brendan Hassett and Donghoon Hyeon. Log minimal model program for the moduli space of stable curves: The first flip, arXiv:0806.3444v1 [math.AG]

[Hau03] Herwig Hauser. The Hironaka theorem on resolution of singularities (or: A proof we always wanted to understand). Bull. Amer. Math. Soc. (N.S.), 40(3):323-403 (electronic), 2003. MR 1978567 (2004d:14009)

[HL07] Donghoon Hyeon and Yongnam Lee. Stability of tri-canonical curves of genus two. Math. Ann., 337(2):479-488, 2007. MR2262795 (2007k:14052)

[Mor08] Donghoon Hyeon and Ian Morrison. Stability of tails and 4-canonical models, arXiv:0806.1269v1 [math.AG]

[KT06] Sean Keel and Jenia Tevelev. Geometry of Chow quotients of Grassmannians. Duke Math. J., 134(2):259-311, 2006. MR2248832 (2007m:14017)

[KM98] János Kollár and Shigefumi Mori. Birational geometry of algebraic varieties, volume 134 of Cambridge Tracts in Mathematics. Cambridge University Press, Cambridge, 1998. With the collaboration of C. H. Clemens and A. Corti; Translated from the 1998 Japanese original. MR.1658959 (2000b:14018)

[LMB00] Gérard Laumon and Laurent Moret-Bailly. Champs algébriques, volume 39 of Ergebnisse der Mathematik und ihrer Grenzgebiete. 3. Folge. A Series of Modern Surveys in Mathematics. Springer-Verlag, Berlin, 2000. MR1771927(2001f:14006)

[Mum77] David Mumford. Stability of projective varieties. Enseignement Math. (2), 23(1-2):39110, 1977. MR0450272 (56:8568)

[Ray72] Michel Raynaud. Flat modules in algebraic geometry. Compositio Math., 24:11-31, 1972. MR0302645 (46:1789)

[SB06] Nicholas I. Shepherd-Barron. Perfect forms and the moduli space of abelian varieties. Invent. Math., 163(1):25-45, 2006. MR2208417(2007e:14070)

[Sch91] David Schubert. A new compactification of the moduli space of curves. Compositio Math., 78(3):297-313, 1991. MR1106299 (92d:14018)

Department of Mathematics, Rice University, 6100 Main St., Houston, Texas 772511892

E-mail address: hassett@math.rice.edu

Department of Mathematics, Northern Illinois University, DeKalb, Illinois 60115

E-mail address: hyeon@math.niu.edu

Current address: Department of Mathematics, Marshall University, One John Marshall Drive, Huntington, West Virginia 25755

E-mail address: hyeond@marshall.edu 